\title{
On non-negative modeling with CARMA processes
}

\author{
Fred Espen Benth ${ }^{\mathrm{a}}$, Victor Rohde $\mathrm{b}^{\mathrm{b}, *}$ \\ ${ }^{a}$ Department of Mathematics, University of Oslo, N-0316 Blindern, Norway \\ ${ }^{b}$ Department of Mathematics, Aarhus University, Ny Munkegade 118, 8000 Aarhus C, \\ Denmark
}

\begin{abstract}
Two stationary and non-negative processes that are based on continuoustime autoregressive moving average (CARMA) processes are discussed. First, we consider a generalization of Cox-Ingersoll-Ross (CIR) processes. Next, we consider CARMA processes driven by compound Poisson processes with exponential jumps which are generalizations of Ornstein-Uhlenbeck (OU) processes driven by the same noise. The way in which the two processes generalize CIR and OU processes and the relation between them will be discussed. Furthermore, the stationary distribution, the autocorrelation function, and pricing of zero-coupon bonds are considered.

Keywords: Continuous-time ARMA processes, Lévy processes,

Ornstein-Uhlenbeck processes, Stationary processes, Square-root process

2010 MSC: 60G10, 91G30
\end{abstract}

\section{Introduction}

The CIR process has been studied extensively and found many applications, in particular, as a model for the volatility of a financial asset (known as the Heston volatility model [24]) and as a model for spot interest rates (see [19]). More recently, a CIR process has been proposed to model wind speeds in [5] where a significant reduction in the mean-square prediction error is obtained compared with more classical static models. The OU process is another extensively studied

\footnotetext{
* Corresponding author

Email address: victor@math.au.dk (Victor Rohde)
}

Preprint submitted to Journal of Mathematical Analysis and ApplicationsDecember 18, 2018 
process that is popular in modeling volatility (see [35]), interest rates (known as the Vasicek model [38]), and wind (see [8]). Both the CIR and OU processes have the desirable trait of being continuous-time processes that are relatively simple, analytically tractable, stationary and, when the OU process is driven by a subordinator, non-negative. On the other hand, a drawback of CIR and OU processes is that the correlation structure is not very flexible; the autocorrelation function (ACF) is simply an exponential function. This often results in models that do not capture some of the correlation structure. For example, in their application of a CIR process to wind, we see in [5, Figure 1] (see also Figures 1 and 2 in this paper) that the model ACF overshoots the population ACF for small lags and undershoots it for large lags. This motivates studying non-negative and stationary processes that generalize CIR and OU processes but with a correlation structure that is more flexible.

A natural generalization of OU processes is CARMA processes. CARMA processes are stationary, there are conditions for non-negativity (see [15, 37]), and they have a more flexible correlation structure (cf. Proposition 4.1). Many results about CARMA processes are given in the literature, for example, a prediction formula, a multivariate extension, noise recovery, an extension to Hilbert space valued processes, an extension to incorporate long memory, and a $\operatorname{CAR}(\infty)$ representation (see [4, 9, 14, 15, 17, 30]). Moreover, there exists a large body of literature on the application of CARMA processes. For example, CARMA processes have been used to model realized volatility, interest rates, electricity prices, and temperature (see [1, 6, 7, 13, 15, 22, 36]).

CIR and OU processes are closely related in two ways. First, sums of independent copies of squared Gaussian OU processes constitute special cases of CIR processes. Second, both a CIR process and an OU process driven by a compound Poisson process with exponentially distributed jumps have a gamma distribution as their stationary distribution and an exponential function as their ACF. In this paper we discuss two stationary and non-negative models based on CARMA processes that build on these two connections between CIR and OU processes. 
Firstly, we consider a sum of independent copies of squared Gaussian CARMA processes which, motivated by the discussion above, we will call a CIR-CARMA process. Using a connection between the sum of independent copies of squared CARMA processes and Wishart processes, we are able to extend this class of non-negative stationary processes that naturally generalizes CIR processes.

Secondly, we will discuss a CARMA process driven by a compound Poisson process with exponential jumps. This model is very tractable but its stationary distribution is not well understood. We show that, under assumptions frequently satisfied in practice, the stationary distribution is an infinite sum of independent gamma distributed random variables.

In both models we also discuss the ACF, which we will compare with the ACF of a CIR process (or, equivalently, an OU process), and we price zerocoupon bonds when the spot interest rate is governed by the models to show their analytical tractability.

We start with Section 2 where we briefly introduce the setup. Section 3 introduces the CIR-CARMA model and Section 4 discusses CARMA processes with a focus on when the Lévy process is a compound Poisson process with exponential jumps.

\section{Preliminaries}

Let $(\Omega, \mathcal{F}, \mathbb{P})$ be a complete probability space, equipped with a filtration $\left(\mathcal{F}_{t}\right)_{t \geq 0}$ satisfying the usual hypotheses (complete and right-continuous).

We now introduce CARMA processes. We will only consider causal CARMA processes that is, where the auto-regressive polynomial $P$ has roots with negative real part, and we therefore limit our discussion to those. For a more thorough discussion see for example [12]. For $p \in \mathbb{N}, q \in \mathbb{N} \cup\{0\}$, consider two polynomials $P$ and $Q$ given by

$$
\begin{aligned}
& P(z)=z^{p}+a_{1} z^{p-1}+\cdots+a_{p} \quad \text { and } \\
& Q(z)=b_{0}+b_{1} z+\cdots+b_{q-1} z^{q-1}+z^{q}
\end{aligned}
$$


where $p>q$, and assume that the roots of $P$ have negative real part. Then a causal CARMA $(p, q)$ process $(Y(t))_{t \in \mathbb{R}}$ satisfies the formal differential equation

$$
P(D) Y(t)=Q(D) D L(t)
$$

where $(L(t))_{t \in \mathbb{R}}$ is a two-sided square integrable Lévy process. In the case $(L(t))_{t \in \mathbb{R}}$ is a standard Brownian motion, we denote it by $(B(t))_{t \in \mathbb{R}}$. CARMA processes may be defined for Lévy processes possessing only finite log-moments (see [16]) but, for convenience, we restrict ourselves to the square integrable case. The representation (2.1) can be made rigorous by considering the statespace representation. In particular, let $(X(t))_{t \in \mathbb{R}}$ be a $p$-dimensional OrnsteinUhlenbeck process that satisfies

$$
d X(t)=A X(t) d t+e_{p} d L(t)
$$

where $e_{p}$ is the $p$-th standard basis vector in $\mathbb{R}^{p}$ and

$$
A=\left[\begin{array}{ccccc}
0 & 1 & 0 & \cdots & 0 \\
0 & 0 & 1 & \cdots & 0 \\
\vdots & \vdots & \ddots & \ddots & \vdots \\
0 & 0 & \cdots & 0 & 1 \\
-a_{p} & -a_{p-1} & \cdots & -a_{2} & -a_{1}
\end{array}\right] .
$$

Then $(Y(t))_{t \in \mathbb{R}}$ is defined by $Y(t)=b^{\top} X(t)$ where

$$
b=\left(b_{0}, \ldots, b_{q-1}, 1,0, \ldots, 0\right)^{\top} \in \mathbb{R}^{p},
$$

and $x^{\top}$ denotes the transpose of $x$. We call $(X(t))_{t \in \mathbb{R}}$ the state-space process associated to the CARMA process $(Y(t))_{t \in \mathbb{R}}$.

CARMA processes have a moving average representation, that is,

$$
Y(t)=\int_{\mathbb{R}} g(t-u) d L(u), \quad t \in \mathbb{R},
$$

where $g: \mathbb{R} \rightarrow \mathbb{R}$ is characterized by

$$
\mathcal{F}[g](y):=\int_{\mathbb{R}} e^{-i y x} g(x) d x=\frac{Q(i y)}{P(i y)}, \quad y \in \mathbb{R} .
$$


If $(Y(t))_{t \in \mathbb{R}}$ is causal then $g(x)=b^{\top} e^{A x} e_{p}$ for $x \geq 0$ and zero otherwise.

If the polynomial $P$ has distinct roots with negative real part then the spectral decomposition of $g$ takes the simple form

$$
g(x)=\sum_{i=1}^{p} \frac{Q\left(\alpha_{i}\right)}{P^{\prime}\left(\alpha_{i}\right)} e^{\alpha_{i} x}, \quad x \geq 0,
$$

where $\alpha_{1}, \ldots, \alpha_{p}$ are the roots of $P$ and $P^{\prime}$ denotes the differential of $P$. In general, if $P$ does not have distinct roots, $g$ still has a spectral decomposition but, to remain brief, we will not give the details here (see [12] for more on this case).

We will say a random variable has an exponential distribution with parameter $\kappa>0$ if it has density

$$
x \mapsto \kappa e^{-\kappa x}, \quad x>0,
$$

and say it has a gamma distribution with shape $\alpha>0$ and rate $\beta>0$ if it has density

$$
x \mapsto \frac{\beta^{\alpha}}{\Gamma(\alpha)} x^{\alpha-1} e^{-\beta x}, \quad x>0 .
$$

Here, $\Gamma$ denotes the gamma function.

\section{The CIR-CARMA process}

A sum of independent copies of squared OU processes constitutes a special case of a CIR process. In this section we will investigate what happens if the OU processes are replaced by CARMA processes. In particular, we will connect a sum of independent copies of squared CARMA processes to the Wishart process, which is an extension of CIR processes to the matrix valued case. As we will see, the Wishart process will play a similar role to a sum of independent copies of squared CARMA processes as the state-space process does for CARMA processes.

We start by introducing CIR processes. A process $(r(t))_{t \geq 0}$ is said to be a CIR process if it has dynamics given by

$$
d r(t)=a(b-r(t)) d t+\sigma \sqrt{r(t)} d B(t), \quad r(0)=r_{0} \geq 0,
$$


where $a, b, \sigma>0$ are constants and $(B(t))_{t \in \mathbb{R}}$ is a standard Brownian motion. The process $(r(t))_{t \geq 0}$ is mean-reverting and non-negative (even positive under the Feller condition $2 a b \geq \sigma^{2}$ ).

Another extensively studied mean-reverting stochastic process is the OU process (see, for example, [2, 34]). We say $(X(t))_{t \geq 0}$ is a Gaussian OU process with mean-reversion $\lambda>0$ and volatility $\sigma_{O U}^{2}>0$ if

$$
d X(t)=-\lambda X(t) d t+\sigma_{O U} d B(t), \quad X(0)=X_{0} \in \mathbb{R} .
$$

The stationary distribution of $(X(t))_{t \geq 0}$ is a mean zero Gaussian distribution with variance $\sigma_{O U}^{2} / 2 \lambda$. The following connection between CIR and Gaussian OU processes is well-known (see, for example, [23, Proposition 4] or [26, Chapter 6]).

Proposition 3.1. Let $\left(X_{i}(t)\right)_{t \in \mathbb{R}}, i=1, \ldots, n$, be OU processes driven by independent standard Brownian motions $\left(B_{i}(t)\right)_{t \in \mathbb{R}}, i=1, \ldots, n$, all with meanreversion $\lambda>0$ and volatility $\sigma_{O U}^{2}>0$. Then $r(t)=\sum_{i=1}^{n} X_{i}^{2}(t)$ is a CIR process of the form in (3.1) with

$$
a=2 \lambda, \quad b=\frac{n \sigma_{O U}^{2}}{2 \lambda}, \quad \text { and } \quad \sigma=2 \sigma_{O U} .
$$

Remark 3.2. From Proposition 3.1 we see that not all CIR processes are squared OU processes. In particular, for $a$ and $\sigma$ fixed, $\lambda$ and $\sigma_{O U}$ are determined. Then $b$ needs to be an integer times $\frac{\sigma_{O U}^{2}}{2 \lambda}$ for the CIR process to be a squared OU process. A CIR process makes sense for general $b$, and, in this way, CIR processes generalize sums of squared OU processes.

Instead of summing independent copies of squared Gaussian OU processes as in Proposition 3.1 we will now consider a sum of independent copies of squared causal Gaussian CARMA processes. Let $n \in \mathbb{N}$ and $\left(Y_{i}(t)\right)_{t \in \mathbb{R}}, i=1, \ldots, n$, be independent copies of a causal $\operatorname{CARMA}(p, q)$ process driven by $\left(\sigma B_{i}(t)\right)_{t \in \mathbb{R}}$, $i=1, \ldots, n$, for $\sigma>0$, where $\left(B_{i}(t)\right)_{t \in \mathbb{R}}, i=1, \ldots, n$ are independent standard Brownian motions, and let $\left(X_{i}(t)\right)_{t \in \mathbb{R}}, i=1, \ldots, n$ be the associated state-space processes. Finally, we define the process $(C(t))_{t \in \mathbb{R}}$ by

$$
C(t)=\sum_{i=1}^{n} Y_{i}^{2}(t)=b^{\top} Z(t) b
$$


where $(Z(t))_{t \in \mathbb{R}}$ is given by

$$
Z(t)=\sum_{i=1}^{n} X_{i}(t) X_{i}^{\top}(t)
$$

We will say that $(C(t))_{t \in \mathbb{R}}$ is a $\operatorname{CIR-CARMA}(p, q)$ process.

The following proposition is a direct consequence of the definition of $(C(t))_{t \in \mathbb{R}}$ but we still highlight the result since it is an important point for the model.

Proposition 3.3. Let $(C(t))_{t \in \mathbb{R}}$ be given by 3.2 . Then $C(t)$ is gamma distributed with scale $n / 2$ and rate $\left(2 \sigma^{2}\|g\|_{2}^{2}\right)^{-1}$, where $g: \mathbb{R} \rightarrow \mathbb{R}$ is given by

$$
g(x)=b^{\top} e^{A x} e_{p}, \quad x \geq 0,
$$

and zero otherwise, and $\|\cdot\|_{2}$ is the $L^{2}(\mathbb{R})$-norm.

Proof. It follows by the moving average representation of a CARMA process in (2.4) that $Y_{i}(t) /\left(\sigma\|g\|_{2}\right)$ has a standard Gaussian distribution. Since

$$
C(t)=\sigma^{2}\|g\|_{2}^{2} \sum_{i=1}^{n}\left(\frac{Y_{i}(t)}{\sigma\|g\|_{2}}\right)^{2}
$$

we therefore have that $C(t)$ is the product of $\sigma^{2}\|g\|_{2}^{2}$ and a chi-square distributed variable with $n$ degrees of freedom, or, equivalently, $C(t)$ is gamma distributed with scale $n / 2$ and rate $\left(2 \sigma^{2}\|g\|_{2}^{2}\right)^{-1}$.

We now state the ACF of a CIR-CARMA process.

Proposition 3.4. Let $(C(t))_{t \in \mathbb{R}}$ be given by 3.2 . Then, for $s \leq t$,

$$
\operatorname{corr}(C(t), C(s))=\left(\frac{b^{\top} e^{A(t-s)} \int_{0}^{\infty} e^{A u} e_{p} e_{p}^{\top} e^{A^{\top} u} d u b}{\int_{0}^{\infty}\left(b^{\top} e^{A u} e_{p}\right)^{2} d u}\right)^{2} .
$$

Proof. Initially, we note that for any mean zero two-dimensional Gaussian variable $\left(G_{1}, G_{2}\right)$ it follows from Isserlis' Theorem (cf. 25]) that

$$
\mathbb{E}\left[G_{1}^{2} G_{2}^{2}\right]=\mathbb{E}\left[G_{1}^{2}\right] \mathbb{E}\left[G_{2}^{2}\right]+2\left(\mathbb{E}\left[G_{1} G_{2}\right]\right)^{2},
$$

and therefore

$$
\begin{aligned}
\operatorname{cov}\left(G_{1}^{2}, G_{2}^{2}\right) & =\mathbb{E}\left[G_{1}^{2} G_{2}^{2}\right]-\mathbb{E}\left[G_{1}^{2}\right] \mathbb{E}\left[G_{2}^{2}\right] \\
& =2\left(\mathbb{E}\left[G_{1} G_{2}\right]\right)^{2} .
\end{aligned}
$$


Next note that we may assume $n=1$ in 3.2 since $C(t)$ is a sum of independent copies. Then, since $\left(Y_{1}(t)\right)_{t \in \mathbb{R}}$ is a mean zero Gaussian process and

$$
\begin{aligned}
\mathbb{E}\left[Y_{1}(t) Y_{1}(s)\right] & =\mathbb{E}\left[\int_{-\infty}^{t} g(t-u) d B(u) \int_{-\infty}^{s} g(s-u) d B(u)\right] \\
& =\int_{\mathbb{R}} g(t-u) g(s-u) d u \\
& =b^{\top} \int_{0}^{\infty} e^{A(t-s+u)} e_{p} e_{p}^{\top} e^{A^{\top} u} d u b,
\end{aligned}
$$

the result follows.

If the roots of $P$ are distinct, then it is argued in [12, section 3] that

$$
\begin{array}{r}
b^{\top} e^{A(t-s)} \int_{0}^{\infty} e^{A u} e_{p} e_{p}^{\top} e^{A^{\top} u} d u b \\
=\sum_{i=1}^{p} \frac{Q\left(\alpha_{i}\right) Q\left(-\alpha_{i}\right)}{P^{\prime}\left(\alpha_{i}\right) P\left(-\alpha_{i}\right)} e^{\alpha_{i}(t-s)}
\end{array}
$$

where $\alpha_{1}, \ldots, \alpha_{p}$ are the roots of $P$, which simplifies calculating the correlation given in Proposition 3.4

In Figure 1 the population $\mathrm{ACF}$ for the wind speeds at the turbine rotor height every 10 minutes over 365 days in 2005 (ID 24500 located $43.48 \mathrm{~N}$ and 107.29W in Wyoming, USA) is plotted 1 We also plot the ACFs of the CIR and CIR-CARMA $(2,1)$ that minimize the squared distance between the 10 minutes lags for a total of 4 days. A similar plot can be found in [5, Figure 1] where the population $\mathrm{ACF}$ is compared with the $\mathrm{CIR} \mathrm{ACF}$ calibrated using maximum likelihood estimation on the same data. The CIR process given by 3.1 has $\mathrm{ACF}$

$$
t \mapsto e^{-a t}, \quad t \geq 0
$$

(see, for example, [5]). Here, $t$ is measured in units of ten minutes. For the CIR process we find $a=0.0069$ as the calibrated parameter and for the CIR-

\footnotetext{
${ }^{1}$ These data have been made available to us by Alexandre Brouste and corresponds to the same data used in [5]. Unfortunately, these data are not anymore available on wind.nrel.gov as referred to in [5].
} 
CARMA $(2,1)$ process we find the calibrated parameters

$$
\alpha_{1}=-0.0024, \quad \alpha_{2}=-0.0296, \quad \text { and } \quad \beta_{1}=-0.0165 \text {, }
$$

where $\alpha_{1}$ and $\alpha_{2}$ are the roots of $P$ and $\beta_{1}$ is the root of $Q$. The CIR-CARMA process is seen to capture the correlation structure very well and, in particular, overcome the difficulties of the CIR process in both capturing the rapid decay for small lags and slow decay for large lags of the population ACF.

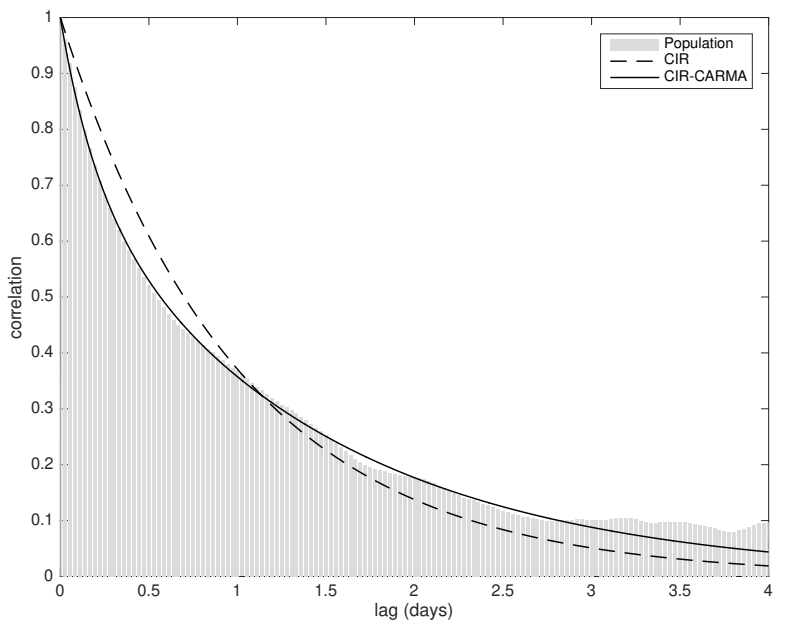

Figure 1: Population ACF in gray, CIR ACF (dotted line), and CIR-CARMA(2,1) ACF (solid line).

Next, to show the analytical tractability of the CIR-CARMA process, we apply it to zero-coupon bond pricing. We assume that the short rate is given by the CIR-CARMA process in (3.2), that is, $r(t)=C(t)$, and where we use $\mathbb{P}$ as the pricing measure. Following the standard theory for pricing based on short rate processes, see e.g. [21], we define the zero-coupon bond price $P(t, T)$ at time $t \geq 0$ for a contract maturing at time $T \geq t$ by

$$
P(t, T)=\mathbb{E}\left[\exp \left\{-\int_{t}^{T} r(s) d s\right\} \mid \mathcal{F}_{t}\right] .
$$

We find the following result: 
Theorem 3.5. Let $(C(t))_{t \in \mathbb{R}}$ and $(Z(t))_{t \in \mathbb{R}}$ be given by 3.2 and $(3.3)$, respectively. Then, for $0 \leq t \leq T<\infty$,

$$
P(t, T)=\exp \{n \phi(T-t)+\operatorname{tr}(\Phi(T-t) Z(t))\},
$$

where tr is the trace operator,

$$
\Phi(t)=\Phi_{1}(t) \Phi_{2}(t)^{-1}
$$

with $\Phi_{1}: \mathbb{R} \rightarrow \mathbb{R}^{p \times p}, \Phi_{2}: \mathbb{R} \rightarrow \mathbb{R}^{p \times p}$, and $\phi: \mathbb{R} \rightarrow \mathbb{R}$ given by

$$
\left(\begin{array}{c}
\Phi_{1}(t) \\
\Phi_{2}(t)
\end{array}\right)=\exp \left(\left(\begin{array}{cc}
A^{\top} & -b b^{\top} \\
-2 \sigma^{2} e_{p} e_{p}^{\top} & -A
\end{array}\right) t\right)\left(\begin{array}{c}
0_{p} \\
I_{p}
\end{array}\right)
$$

and

$$
\phi(t)=\sigma^{2} \int_{0}^{t} e_{p}^{\top} \Phi(s) e_{p} d s
$$

Here, $I_{p}$ is the $p \times p$ identity matrix and $0_{p}$ is the matrix in $\mathbb{R}^{p \times p}$ of zeros.

Proof. Initially, we note that, by independence, we may assume $n=1$ in 3.2 . For $u:[0, \infty) \times \mathbb{R}^{p} \rightarrow \mathbb{R}$, consider the parabolic partial differential equation

$$
\begin{aligned}
\partial_{t} u(t, x) & =\frac{1}{2} \sigma^{2} \partial_{p}^{2} u(t, x)+\nabla_{x} u(t, x) A x-\left(b^{\top} x\right)^{2} u(t, x) \\
u(0, x) & =1 .
\end{aligned}
$$

Here, $x \in \mathbb{R}^{p}, t \geq 0$, and $\partial_{p}$ denotes the differentiation with respect to the $p^{\prime}$ th entry of $x$. Assume that the solution $u$ is given by

$$
u(t, x)=\exp \left\{\phi(t)+x^{\top} \Phi(t) x\right\}
$$

Then

$$
\begin{aligned}
\partial_{t} u(t, x) & =\left(\phi^{\prime}(t)+x^{\top} \Phi^{\prime}(t) x\right) u(t, x) \\
\nabla_{x} u(t, x) & =x^{\top}\left(\Phi(t)+\Phi^{\top}(t)\right) u(t, x) \\
\partial_{p}^{2} u(t, x) & =\left(2 e_{p}^{\top} \Phi(t) e_{p}+x^{\top}\left(\Phi(t)+\Phi^{\top}(t)\right) e_{p} e_{p}^{\top}\left(\Phi(t)+\Phi^{\top}(t)\right) x\right) u(t, x),
\end{aligned}
$$


and it therefore follows that $u$ solves 3.10 if $\Phi$ is symmetric, and $\phi$ and $\Phi$ solves

$$
\begin{aligned}
\phi^{\prime}(t) & =\sigma^{2} e_{p}^{\top} \Phi(t) e_{p}, \\
\phi(0) & =0, \\
\Phi^{\prime}(t) & =2 \sigma^{2} \Phi(t) e_{p} e_{p}^{\top} \Phi(t)+\Phi(t) A+A^{\top} \Phi(t)-b b^{\top}, \\
\Phi(0) & =0_{p}, \quad t \geq 0 .
\end{aligned}
$$

Here, we have used that $x^{\top} 2 \Phi^{\top} A x=x^{\top} A^{\top} \Phi x+x^{\top} \Phi^{\top} A x$. Given $\Phi, \phi$ is readily found by integration. To find $\Phi$ we recognize the equation as a matrix Riccati equation (see for example [28] where a simple change of variable is necessary since the Riccati equation is considered backward). It follows from [28, Theorem 8] that 3.12 has a unique solution on $[0, \infty)$. Since the transpose of a solution again is a solution, it is symmetric. By [28, Theorem 9] we have that the solution is negative semi-definite and therefore that $u$ of the form in (3.11) is bounded and, in particular, of at most polynomial growth. Furthermore, by [29], the solution is given by (3.8) and (3.9).

By the Feynman-Kac formula (see for example [27, Theorem 7.6, Chapter 5]), the solution $u$ is unique with representation

$$
\mathbb{E}\left[\exp \left\{-\int_{t}^{T}\left(b^{\top} X(s)\right)^{2} d s\right\} \mid X(t)=x\right]=u(T-t, x) .
$$

Since

$$
\begin{aligned}
\mathbb{E}\left[\exp \left\{-\int_{t}^{T} r(s) d s\right\} \mid \mathcal{F}_{t}\right] & =\mathbb{E}\left[\exp \left\{-\int_{t}^{T}\left(b^{\top} X(s)\right)^{2} d s\right\} \mid \mathcal{F}_{t}\right] \\
& \left.=\mathbb{E}\left[\exp \left\{\phi(t)+X(t)^{\top} \Phi(t) X(t)\right)\right\} \mid \mathcal{F}_{t}\right] \\
& =\exp \{\phi(t)+\operatorname{tr}(\Phi(t) Z(t))\}
\end{aligned}
$$

the result now follows.

Some comments are in place. Firstly, by considering the case $p=1$ where we are back to the classical CIR process, it is simple to see that Theorem 3.5 reduces 
to the known zero-coupon bond price for CIR short rate models (see [19]). Next, in mathematical finance, one usually differentiates between the market probability and pricing measures, sometimes called equivalent martingale measures, denoted $\mathbb{Q} \sim \mathbb{P}$. In the theorem above, we simply used $\mathbb{Q}=\mathbb{P}$. We may, however, assume that the CIR-CARMA model is formulated under some $\mathbb{Q} \sim \mathbb{P}$ directly, and obtain the price for $P(t, T)$ under a conditional expectation with respect to $\mathbb{Q}$. We obtain, of course, the price as in Theorem 3.5. The market prices of $P(t, T)$ is, on the other hand, observed under the market probability $\mathbb{P}$, and to have its dynamics under $\mathbb{P}$ we must specify the $\mathbb{P}$-dynamics of $(Z(t))_{t \in \mathbb{R}}$. As we have a Brownian-based model for $(Z(t))_{t \in \mathbb{R}}$, this can be done by referring to Girsanov's Theorem. Further, we note that the zero-coupon bond price is represented in terms of the process $(Z(t))_{t \in \mathbb{R}}$, which is not directly observable. This is contrary to the CIR model, where the price is explicit in terms of the current state of the interest rate $r(t)$. To make inference, say filtering techniques are called for in the general CIR-CARMA case.

We now connect the CIR-CARMA model to Wishart processes. This will allow us do define more general processes that still are generalizations of CIR processes. The main idea of the proof is to connect multivariate Gaussian OU processes to a Wishart process. This is also discussed in [18].

Theorem 3.6. Let $(C(t))_{t \in \mathbb{R}}$ be defined as in 3.2 and assume $n \geq p$. Then

$$
C(t)=b^{\top} Z(t) b
$$

where $(Z(t))_{t \in \mathbb{R}}$ is an $p \times p$ dimensional process with dynamics

$$
\begin{aligned}
d Z(t) & =\left(n \sigma^{2} e_{p} e_{p}^{\top}+A Z(t)+Z(t) A^{\top}\right) d t \\
& +Z(t)^{1 / 2} d W(t) \sigma e_{p} e_{p}^{\top}+\sigma e_{p} e_{p}^{\top} d W(t)^{\top} Z(t)^{1 / 2},
\end{aligned}
$$

and $(W(t))_{t \in \mathbb{R}}$ is a $p \times p$ dimensional standard matrix Brownian motion, that is, the entries consist of independent standard Brownian motions.

Proof. We have

$$
\sum_{i=1}^{n} Y_{i}(t)^{2}=b^{\top}\left(\sum_{i=1}^{n} X_{i}(t) X_{i}(t)^{\top}\right) b .
$$


By the multivariate version of Ito's lemma (see for example [32, Theorem 4.2.1])

$$
\begin{aligned}
d\left(X_{i}(t) X_{i}(t)^{\top}\right)= & X_{i}(t) d X_{i}(t)^{\top}+\left(d X_{i}(t)\right) X_{i}(t)^{\top}+d X_{i}(t) d X_{i}(t)^{\top} \\
= & \left(X_{i}(t) X_{i}(t)^{\top} A^{\top}+A X_{i}(t) X_{i}(t)^{\top}+\sigma^{2} e_{p} e_{p}^{\top}\right) d t \\
& +\left(X_{i}(t) e_{p}^{\top}+e_{p} X_{i}(t)^{\top}\right) \sigma d B_{i}(t) .
\end{aligned}
$$

Since $n \geq p, \sum_{i=1}^{n} X_{i}(s) X_{i}(s)^{\top}$ is positive definite almost surely. Let

$$
\tilde{W}(t)=\int_{0}^{t}\left(\sum_{i=1}^{n} X_{i}(s) X_{i}(s)^{\top}\right)^{-1 / 2} \sum_{i=1}^{n} X_{i}(s) e_{p}^{\top} d B_{i}(s),
$$

where we have used the notation $A^{-1 / 2}=\left(A^{1 / 2}\right)^{-1}$ for a positive definite matrix $A$. Denote by $[\cdot, \cdot]$ the quadratic covariation and by $B_{i, j}$ the $(i, j)$ 'th entry of a $p \times p$ matrix $B$. Consider $1 \leq i_{1}, i_{2}, j_{1}, j_{2} \leq p$. Then

$$
\begin{aligned}
{\left[\tilde{W}_{i_{1}, j_{1}}, \tilde{W}_{i_{2}, j_{2}}\right](t)=} & \sum_{i=1}^{n} \int_{0}^{t}\left(\left(\sum_{i=1}^{n} X_{i}(s) X_{i}(s)^{\top}\right)^{-1 / 2} X_{i}(s) e_{p}^{\top}\right)_{i_{1}, j_{1}} \\
& \times\left(\left(\sum_{i=1}^{n} X_{i}(s) X_{i}(s)^{\top}\right)^{-1 / 2} X_{i}(s) e_{p}^{\top}\right)_{i_{2}, j_{2}} d s .
\end{aligned}
$$

If either $j_{1}$ or $j_{2}$ is not equal to $p$ then 3.15 is zero. Furthermore,

$$
\begin{aligned}
& \sum_{i=1}^{n}\left(\left(\sum_{i=1}^{n} X_{i}(s) X_{i}(s)^{\top}\right)^{-1 / 2} X_{i}(s) e_{p}^{\top}\right)_{i_{1}, p} \\
& \quad \times\left(\left(\sum_{i=1}^{n} X_{i}(s) X_{i}(s)^{\top}\right)^{-1 / 2} X_{i}(s) e_{p}^{\top}\right)_{i_{2}, p} \\
& =\sum_{i=1}^{n}\left(\left(\sum_{i=1}^{n} X_{i}(s) X_{i}(s)^{\top}\right)^{-1 / 2} X_{i}(s) X_{i}(s) \top\left(\sum_{i=1}^{n} X_{i}(s) X_{i}(s)^{\top}\right)^{-1 / 2}\right)_{i_{1}, i_{2}} \\
& =\left(I_{p}\right)_{i_{1}, i_{2}} .
\end{aligned}
$$

It follows that $(\tilde{W}(t))_{t \in \mathbb{R}}$ is a $p \times p$ dimensional Brownian motion by Lévy's characterization of Brownian motion (see for example [32, Theorem 8.6.1]) of the form $W(t) e_{p} e_{p}^{\top}$, where $(W(t))_{t \in \mathbb{R}}$ is a $p \times p$ standard matrix Brownian 
motion, that is, the entries of $(W(t))_{t \in \mathbb{R}}$ are independent standard Brownian motions. Let $Z(t)$ be given by (3.3). We then conclude that

$$
\begin{aligned}
d Z(t) & =\left(n \sigma^{2} e_{p} e_{p}^{\top}+A Z(t)+Z(t) A^{\top}\right) d t \\
& +Z(t)^{1 / 2} d W(t) \sigma e_{p} e_{p}^{\top}+\sigma e_{p} e_{p}^{\top} d W(t)^{\top} Z(t)^{1 / 2}
\end{aligned}
$$

and the proof is complete.

It is natural to generalize the level $n \sigma^{2} e_{p} e_{p}^{\top}$ in the dynamics of $(Z(t))_{t \geq 0}$ given in (3.14) to a general level as a direct parallel to the way sums of squared OU processes can be generalized by a CIR process to accommodate a general level. Thus, we are interested in finding a solution to

$$
\begin{aligned}
d Z(t)=( & \left.\kappa e_{p} e_{p}^{\top}+A Z(t)+Z(t) A^{\top}\right) d t \\
& +Z(t)^{1 / 2} d W(t) \sigma e_{p} e_{p}^{\top}+\sigma e_{p} e_{p}^{\top} d W(t)^{\top} Z(t)^{1 / 2}, \quad Z(0)=Z_{0}
\end{aligned}
$$

for some general $\kappa$ and positive semi-definite $p \times p$ matrix $Z_{0}$. Such processes are Wishart processes and have been studied in [18, 20, 31. In particular, in [20], it is argued that whenever $\kappa \geq(p-1) \sigma^{2}$ then there exists an affine Markov process on the space of positive semi-definite matrices with the dynamics in (3.16). We are led to extending the class of $\operatorname{CIR-CARMA}(p, q)$ processes to be a process $(C(t))_{t \in \mathbb{R}}$ of the form

$$
C(t)=b^{\top} Z(t) b
$$

where $b$ is given in 2.3$), A$ is given in 2.2 , and where $\left(Z_{t}\right)_{t \geq 0}$ is the affine Markov process given in [20] with dynamics given by 3.16 .

The definition of a CIR-CARMA process has a similar structure to that of a CARMA process. In particular, it is defined as a linear transformation of a higher dimensional process. It therefore seems natural to consider the process $(Z(t))_{t \geq 0}$ as a state-space process associated to the CIR-CARMA process $(C(t))_{t \geq 0}$.

It follows immediately from the definition of $b$ and the CIR-CARMA $(p, q)$ process that its paths are $p-q-1$ times continuously differentiable. This is a direct parallel to CARMA processes where a similar statement holds. 
If we have $\kappa \geq(p+1) \sigma^{2}$ in (3.16) then [31] shows that there is a strong unique solution, and it is argued that if, additionally, $Z_{0}$ is positive definite then $Z(t)$ remains positive definite for all $t \geq 0$. This result may be regarded as a version of the Feller condition for Wishart processes. We summarize in the following Proposition:

Proposition $3.7([31])$. Let $(C(t))_{t \geq 0}$ be a CIR-CARMA $(p, q)$ process and assume that $Z_{0}$ is positive definite and $\kappa \geq(p+1) \sigma^{2}$. Then $C(t)>0$ for all $t \geq 0$.

\section{CARMA processes driven by a subordinator}

In this section we will consider causal CARMA processes as an alternative to CIR-CARMA. We are mainly interested in the case where the background driving Lévy process is a compound Poisson process with exponentially distributed jumps and specifications of the CARMA parameters such that the kernel function is non-negative. In this case, the CARMA process will itself become non-negative.

Modeling with a non-negative CARMA process as opposed to the CIRCARMA discussed above has the advantage of, in many respects, being a simpler non-negative model that still maintains a large class of possible correlation

structures. A downside is, however, that the stationary distribution is more complicated. We address the question of characterizing the stationary distribution under some specific assumptions, but start by considering the ACF and pricing of zero-coupon bonds when the spot interest is modeled by the CARMA process.

The following proposition is well-known (see for example [12, Section 3]).

Proposition 4.1. Let $(Y(t))_{t \in \mathbb{R}}$ be a causal CARMA(p,q) process driven by a square integrable Lévy process. Then

$$
\operatorname{corr}(Y(t), Y(s))=\frac{b^{\top} e^{A(t-s)} \int_{0}^{\infty} e^{A u} e_{p} e_{p}^{\top} e^{A^{\top} u} d u b}{\int_{0}^{\infty}\left(b^{\top} e^{A u} e_{p}\right)^{2} d u}, \quad s \leq t .
$$


Remark 4.2. The above result should be compared with Proposition 3.4 . Furthermore, 3.5 may be applied to easily calculate the ACF if the roots of $P$ are distinct.

We again compare the correlation structure of a CARMA $(2,1)$ with that of a CIR process when calibrated to the wind data described in Section 3 . The resulting correlations are given in Figure 2, The calibrated CARMA parameters are

$$
\alpha_{1}=-0.0049, \quad \alpha_{2}=-0.0339, \quad \text { and } \quad \beta_{1}=-0.0179 \text {, }
$$

where $\alpha_{1}$ and $\alpha_{2}$ are the roots of $P$ and $\beta_{1}$ is the root of $Q$. Appealing to [37, these parameters corresponds to a non-negative CARMA kernel.

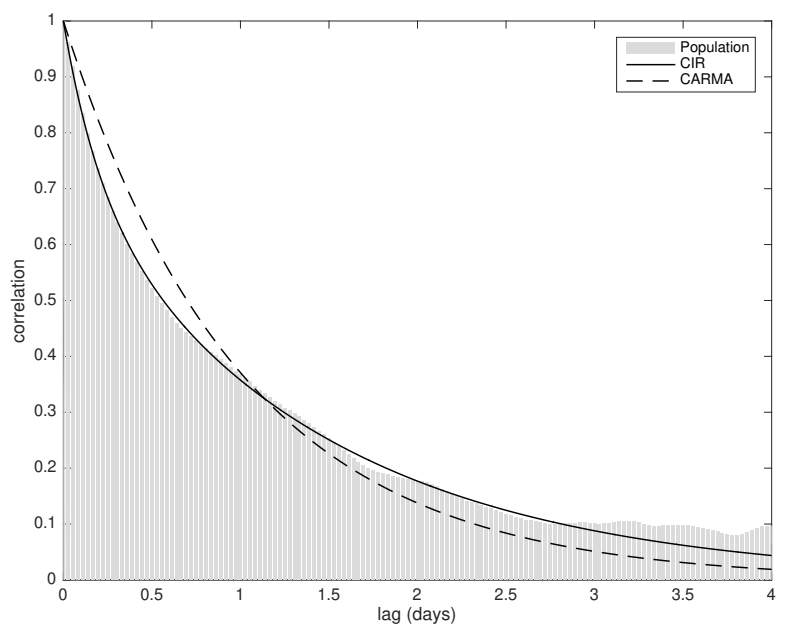

Figure 2: Population acf in gray, CIR ACF (dotted line), and CARMA(2,1) ACF (solid line).

For comparison with the CIR-CARMA case, we give the zero-coupon bond price when the short rate is modeled as a $\operatorname{CARMA}(p, q)$ process.

Proposition 4.3. Let $(Y(t))_{t \in \mathbb{R}}$ be a causal CARMA(p,q) process driven by a compound Poisson process with intensity $\mu>0$ and exponentially distributed jumps with parameter $\kappa>0$. Assume that the kernel of the CARMA process is 
non-negative. Then

$$
\begin{aligned}
P(t, T):= & \mathbb{E}\left[\exp \left\{-\int_{t}^{T} Y(s) d s\right\} \mid L(t)-L(u), u<t\right] \\
= & \exp \left\{b^{\top} A^{-1}\left(I_{p}-e^{A(T-t)}\right) X(t)\right. \\
& \left.+\mu \int_{0}^{T-t} \frac{b^{\top} A^{-1}\left(I_{p}-e^{A u}\right) e_{p}}{\kappa+b^{\top} A^{-1}\left(e^{A u}-I_{p}\right) e_{p}} d u\right\}
\end{aligned}
$$

where $(X(t))_{t \in \mathbb{R}}$ is the associated state-space process.

Before proving Proposition 4.3 we first give the following expression for the moment generating function of a moving average process, which also will be useful in the proof of Theorem 4.8

Lemma 4.4. Let $f \in L^{1}(\mathbb{R})$ be a right-continuous and non-negative bounded function with support on $[0, \infty)$ and define $d=\sup _{u \geq 0}|f(u)|$. Let $(L(t))_{t \in \mathbb{R}}$ be a compound Poisson process with intensity $\mu>0$ and exponentially distributed jumps with parameter $\kappa>0$. Then

$$
\log \mathbb{E}\left[\exp \left\{x \int_{-\infty}^{t} f(t-u) d L(u)\right\}\right]=\mu \int_{0}^{\infty} \frac{x f(u)}{\kappa-x f(u)} d u
$$

for $x \in(-\infty, \kappa / d)$, and

$$
\int_{0}^{\infty} \frac{x f(u)}{\kappa-x f(u)} d u=\sum_{n=1}^{\infty}\left(\frac{x}{\kappa}\right)^{n} \int_{0}^{\infty} f(u)^{n} d u
$$

for $x \in(-\kappa / d, \kappa / d)$.

Proof. Initially we note that for $s<t$ and any $x \in(-\infty, \kappa)$,

$$
\log \mathbb{E}[\exp \{x(L(t)-L(s))\}]=(t-s) \mu \frac{x}{\kappa-x} .
$$

Since $f$ is right-continuous and non-negative there exists an increasing sequence of piece-wise constant functions $\left(f_{n}\right)_{n \in \mathbb{N}}$ of the form

$$
f_{n}(t)=\sum_{m=0}^{\infty} a_{m, n} \mathbb{1}_{\left[m / 2^{n},(m+1) / 2^{n}\right)}(t)
$$


with $f_{n} \rightarrow f$ which implies that $\int_{\mathbb{R}} f_{n}(t-u) d L(u) \rightarrow \int_{\mathbb{R}} f(t-u) d L(u)$ in probability as $n \rightarrow \infty$ (cf. [33]). Since $(L(t))_{t \in \mathbb{R}}$ is non-decreasing and $f_{n}$ is given by (4.3), $\int_{\mathbb{R}} f_{n}(t-u) d L(u)$ is non-decreasing in $n$. We therefore also have almost sure convergence. Let $x \in(-\infty, \kappa / d)$, and note that

$$
|\kappa-x f(u)| \geq \kappa-|x| d>0
$$

Now it follows from 4.2 and 4.3 that

$$
\begin{aligned}
\log \mathbb{E} & {\left[\exp \left\{x \int_{-\infty}^{t} f(t-u) d L(u)\right\}\right] } \\
& =\lim _{n \rightarrow \infty} \log \mathbb{E}\left[\exp \left\{x \int_{-\infty}^{t} f_{n}(t-u) d L(u)\right\}\right] \\
& =\mu \lim _{n \rightarrow \infty} \int_{0}^{\infty} \frac{x f_{n}(u)}{\kappa-x f_{n}(u)} d u \\
& =\mu \int_{0}^{\infty} \frac{x f(u)}{\kappa-x f(u)} d u
\end{aligned}
$$

where we have used both monotone and dominated convergence. The last statement of the lemma is straightforward.

Proof of Proposition 4.3. Since $(X(t))_{t \in \mathbb{R}}$ is a multivariate OU process,

$$
\int_{t}^{T} X(s) d s=A^{-1}\left(X(T)-X(t)-e_{p}(L(T)-L(t)),\right.
$$

and therefore

$$
\int_{t}^{T} Y(s) d s=\int_{t}^{T} b^{\top} X(s) d s=b^{\top} A^{-1}\left(X(T)-X(t)-e_{p}(L(T)-L(t))\right) .
$$

Note that

$$
X(T)=\int_{t}^{T} e^{A(T-u)} e_{p} d L(u)+e^{A(T-t)} X(t)
$$

and, since the kernel is non-negative,

$$
b^{\top} A^{-1}\left(e^{A t}-I_{p}\right) e_{p}=\int_{0}^{t} b^{\top} e^{A u} e_{p} d u \geq 0 .
$$


Consequently, by Lemma 4.4 , with $f(t)=b^{\top} A^{-1}\left(e^{A t}-I_{p}\right) e_{p}$ and $x=-1$, we conclude that

$$
\begin{aligned}
\mathbb{E}\left[\exp \left\{-\int_{t}^{T} Y(s) d s\right\} \mid L(t)-L(u), u \leq t\right] \\
=\exp \left\{b^{\top} A^{-1}\left(I_{p}-e^{A(T-t)}\right) X(t)\right\} \\
\quad \times \mathbb{E}\left[\exp \left\{-b^{\top} A^{-1}\left(\int_{t}^{T} e^{A(T-u)} e_{p} d L(u)-e_{p}(L(T)-L(t))\right)\right\}\right] \\
=\exp \left\{b^{\top} A^{-1}\left(I_{p}-e^{A(T-t)}\right) X(t)+\mu \int_{0}^{T-t} \frac{b^{\top} A^{-1}\left(I_{p}-e^{A u}\right) e_{p}}{\kappa+b^{\top} A^{-1}\left(e^{A u}-I_{p}\right) e_{p}} d u\right\} .
\end{aligned}
$$

The proof is complete.

The following Corollary follows from Lemma 4.4, and gives a series representation of the cumulant function of $Y$.

Corollary 4.5. Let $(Y(t))_{t \in \mathbb{R}}$ be a causal $\operatorname{CARMA}(p, q)$ process driven by a compound Poisson process with intensity $\mu>0$ and exponentially distributed jumps with parameter $\kappa>0$. Let $d=\sup _{u \geq 0}|g(u)|$. Then

$$
\log \mathbb{E}\left[e^{x Y(t)}\right]=\mu \sum_{n=1}^{\infty} \frac{a_{n}}{n}\left(\frac{x}{\kappa}\right)^{n}, \quad x \in(-\kappa / d, \kappa / d),
$$

where

$$
a_{n}=n \int_{0}^{\infty}\left(b^{\top} e^{A u} e_{p}\right)^{n} d u
$$

Let us consider the case of an OU process:

Example 4.6. Let $(Y(t))_{t \in \mathbb{R}}$ be an OU process (corresponding to the CARMA $(1,0)$ case). Then $A$ is a negative scalar and therefore

$$
a_{n}=n \int_{0}^{\infty} e^{n A u} d u=-\frac{1}{A} .
$$

Consequently, the expression in 4.5 reduces to

$$
\log \mathbb{E}\left[e^{x Y(t)}\right]=-\frac{\mu}{A} \sum_{n=1}^{\infty} \frac{1}{n}\left(\frac{x}{\kappa}\right)^{n}=\frac{\mu}{A} \log \left(1-\frac{x}{\kappa}\right),
$$


which we recognize as the cumulant of the gamma distribution with shape $-\mu / A$ and rate $\kappa$. In particular, by Corollary 4.5, we recover the well-known result that an OU process driven by a compound Poisson process with exponential jumps has the gamma distribution as its stationary distribution (see for example [3]).

We now constrain our analysis to the case of $\operatorname{CARMA}(p, p-1)$ processes.

Lemma 4.7. Let $(Y(t))_{t \in \mathbb{R}}$ be a causal $C A R M A(p, p-1)$ process driven by a compound Poisson process with intensity $\mu>0$ and exponentially distributed jumps with parameter $\kappa>0$. Let $\left(a_{n}\right)_{n \in \mathbb{N}}$ be given by 4.6 and assume

$$
\frac{d}{d u} b^{\top} e^{A u} e_{p}=b^{\top} A e^{A u} e_{p}<0, \quad u>0 .
$$

Then $\left(a_{n}\right)_{n \in \mathbb{N}}$ is a convergent sequence with

$$
a_{1}=-b^{\top} A^{-1} e_{p} \quad \text { and } \quad a_{\infty}:=\lim _{n \rightarrow \infty} a_{n}=-\frac{1}{b^{\top} A e_{p}} .
$$

Proof. Using integration by parts we find that

$$
\begin{aligned}
a_{n} & =n \int_{0}^{\infty}\left(b^{\top} e^{A u} e_{p}\right)^{n} d u \\
& =\int_{0}^{\infty} \frac{d}{d u}\left(\left(b^{\top} e^{A u} e_{p}\right)^{n}\right) \frac{b^{\top} e^{A u} e_{p}}{b^{\top} A e^{A u} e_{p}} d u \\
& =\left[\frac{\left(b^{\top} e^{A u} e_{p}\right)^{n+1}}{b^{\top} A e^{A u} e_{p}}\right]_{0}^{\infty}-\int_{0}^{\infty}\left(b^{\top} e^{A u} e_{p}\right)^{n} \frac{d}{d u} \frac{b^{\top} e^{A u} e_{p}}{b^{\top} A e^{A u} e_{p}} d u \\
& =-\frac{1}{b^{\top} A e_{p}}-\int_{0}^{\infty}\left(b^{\top} e^{A u} e_{p}\right)^{n}\left(1-\frac{b^{\top} e^{A u} e_{p} b^{\top} A^{2} e^{A u} e_{p}}{\left(b^{\top} A e^{A u} e_{p}\right)^{2}}\right) d u .
\end{aligned}
$$

Since the function $u \mapsto b^{\top} e^{A u} e_{p}$ is continuous, converges to zero when $u \rightarrow \infty$, is 1 at $u=0$, and is strictly decreasing on $(0, \infty)$ by assumption, we conclude that $0<b^{\top} e^{A u} e_{p}<1$ for all $u>0$. Thus, by monotone convergence, $\left(a_{n}\right)_{n \in \mathbb{N}}$ is convergent with limit $-\frac{1}{b^{\top} A e_{p}}$. The statement about $a_{1}$ is a simple calculation.

Let $\Gamma(\eta, \theta)$ denote the gamma distribution with rate $\eta$ and scale $\theta$. The following is the main result of this section.

Theorem 4.8. For $p \geq 2$, let $(Y(t))_{t \in \mathbb{R}}$ be a causal $C A R M A(p, p-1)$ process driven by a compound Poisson process with intensity $\mu>0$ and exponentially 
distributed jumps with parameter $\kappa>0$. Let $\alpha_{1}, \ldots, \alpha_{p}$ be the roots of $P$ and assume that they are distinct and real. Furthermore, assume

$$
\frac{Q\left(\alpha_{i}\right)}{P^{\prime}\left(\alpha_{i}\right)}>0, \quad i=1, \ldots, p .
$$

Then $G+\sum_{i=1}^{m} G_{i, m}$ converges to $Y(t)$ in distribution as $m \rightarrow \infty$ where

$$
G \sim \Gamma(\mu \eta, \kappa) \quad \text { and } \quad G_{i, m} \sim \Gamma\left(\mu \eta_{i, m}, \kappa \theta_{i, m}\right)
$$

are independent. Here $\eta=-1 /\left(b^{\top} A e_{p}\right)$, and $\eta_{i, m}>0$ and $\theta_{i, m}>1$, for $i=1, \ldots, m$, are such that

$$
\sum_{i=1}^{m} \frac{\eta_{i, m}}{\theta_{i, m}^{n}} \rightarrow-\int_{0}^{\infty}\left(b^{\top} e^{A u} e_{p}\right)^{n} \frac{d}{d u} \frac{b^{\top} e^{A u} e_{p}}{b^{\top} A e^{A u} e_{p}} d u
$$

as $m \rightarrow \infty$.

Proof. By the spectral decomposition,

$$
b^{\top} e^{A t} e_{p}=\sum_{i=1}^{p} c_{i} e^{\alpha_{i} t}
$$

where $c_{i}=\frac{Q\left(\alpha_{i}\right)}{P^{\prime}\left(\alpha_{i}\right)}>0$. Since the CARMA process is causal, $\alpha_{i}<0, i=1, \ldots, p$, and we conclude that the derivative of $t \mapsto b^{\top} e^{A t} e_{p}$ is negative. It follows that the assumptions of Lemma 4.7 are satisfied. Furthermore,

$$
\begin{gathered}
\Delta \sum_{i=0}^{\infty}\left(b^{\top} e^{A \Delta(i+1 / 2)} e_{p}\right)^{n}\left(\frac{b^{\top} e^{A \Delta i} e_{p}}{b^{\top} A e^{A \Delta i} e_{p}}-\frac{b^{\top} e^{A \Delta(i+1)} e_{p}}{b^{\top} A e^{A \Delta(i+1)} e_{p}}\right) \\
\rightarrow-\int_{0}^{\infty}\left(b^{\top} e^{A u} e_{p}\right)^{n} \frac{d}{d u} \frac{b^{\top} e^{A u} e_{p}}{b^{\top} A e^{A u} e_{p}} d u
\end{gathered}
$$

as $\Delta \rightarrow 0$. From the assumption in 4.8 we have $0<b^{\top} e^{A \Delta(i+1 / 2)} e_{p}<1$, and we find that

$$
\begin{aligned}
\left(b^{\top} A e^{A u} e_{p}\right)^{2} & =\sum_{i=1}^{p} c_{i}^{2} \alpha_{i}^{2} e^{2 \alpha_{i} u}+\sum_{i=1}^{p} \sum_{j>i}^{p} c_{j} c_{i} 2 \alpha_{j} \alpha_{i} e^{\left(\alpha_{j}+\alpha_{i}\right) u} \\
& <\sum_{i=1}^{p} c_{i}^{2} \alpha_{i}^{2} e^{2 \alpha_{i} u}+\sum_{i=1}^{p} \sum_{j>i}^{p} c_{j} c_{i}\left(\alpha_{j}^{2}+\alpha_{i}^{2}\right) e^{\left(\alpha_{j}+\alpha_{i}\right) u} \\
& =b^{\top} e^{A u} e_{p} b^{\top} A^{2} e^{A u} e_{p},
\end{aligned}
$$


which implies $\frac{d}{d u} \frac{b^{\top} e^{A u} e_{p}}{b^{\top} A e^{A u} e_{p}}<0$. Let

$$
\theta_{i, m}=\frac{1}{b^{\top} e^{A \Delta_{m}(i+1 / 2)} e_{p}}
$$

and

$$
\eta_{i, m}=\Delta_{m}\left(\frac{b^{\top} e^{A \Delta_{m} i} e_{p}}{b^{\top} A e^{A \Delta_{m} i} e_{p}}-\frac{b^{\top} e^{A \Delta_{m}(i+1)} e_{p}}{b^{\top} A e^{A \Delta_{m}(i+1)} e_{p}}\right),
$$

for $i=1, \ldots, m$ and a sequence $\left(\Delta_{m}\right)_{m \in \mathbb{N}}$ such that $\Delta_{m} \rightarrow 0$ and $m \Delta_{m} \rightarrow \infty$ as $m \rightarrow \infty$. Then we conclude that 4.9 is satisfied. Now, since convergence of the moment generating function implies convergence in distribution (see, for example, [10, Example 5.5]), the result follows from Corollary 4.5 and (4.7).

Remark 4.9. The condition in (4.8) ensures that the kernel function of the CARMA process is a sum of positive scalars times exponential function by the spectral decomposition in 2.5). This is often the case when $\operatorname{CARMA}(p, p-1)$ processes are calibrated to data, for example, it holds for all the applications of CARMA $(p, p-1)$ processes discussed in the introduction in which the calibrated parameters are reported, that is, for [13, 15, 22, 36]. Additionally, it also holds for the parameters in 4.1.

Remark 4.10. The distributional result in Theorem 4.8 is closely related to generalized gamma convolutions albeit slightly different since the gamma distributed variables are dependent on $m$. A distribution $\mu$ is a generalized gamma convolution if there exists a sequence of independent gamma distributed random variables $\left(G_{i}\right)_{i \in \mathbb{N}}$ such that $\sum_{i=1}^{m} G_{i}$ converges to $\mu$ in distribution as $m \rightarrow \infty$. For more on gamma convolutions see for example [11.

Remark 4.11. From 4.11 and 4.12 in the proof of Theorem 4.8 we have available an explicit expression of shapes and rates of gamma distributions where the sum approximates the stationary distribution of the $\operatorname{CARMA}(p, p-1)$. In practice however, it may be more natural to find coefficients that minimize

$$
\sum_{n=1}^{M}\left|\eta+\sum_{i=1}^{m} \frac{\eta_{i, m}}{\theta_{i, m}^{n}}-a_{n}\right|^{2}
$$


for some $M \in \mathbb{N}$, where $\left(a_{n}\right)_{n \in \mathbb{N}}$ is given by 4.7). This works well for small $m$, but we quickly face an identification problem for large $m$. In such cases, the explicit expression of the approximating coefficients have been found to work well.

We now investigate numerically the approximating sequence of the gamma distribution given in Theorem 4.8. We will consider two CARMA $(2,1)$ processes, the first with

$$
\alpha_{1}=-0.5, \quad \alpha_{2}=-1, \quad \text { and } \quad \beta_{1}=-0.75,
$$

and the second with

$$
\alpha_{1}=-0.5, \quad \alpha_{2}=-2, \quad \text { and } \quad \beta_{1}=-0.75 \text {, }
$$

where $\alpha_{1}$ and $\alpha_{2}$ are the roots of $P$ and $\beta_{1}$ is the $\operatorname{root}$ of $Q$. We will also consider a CARMA $(3,2)$ process with

$$
\alpha_{1}=-0.5, \quad \alpha_{2}=-1, \quad \alpha_{3}=-1.2, \quad \beta_{1}=-0.75, \quad \text { and } \quad \beta_{2}=-1.1,
$$

where $\alpha_{1}, \alpha_{2}$, and $\alpha_{3}$ are the roots of $P$, and $\beta_{1}$ and $\beta_{2}$ are the roots of $Q$. The compound Poisson process has intensity $\mu=1$ and the exponential jumps have parameter $\kappa=1$ in all three CARMA models. A simulation of the stationary distributions together with the approximating sum of gamma distributions are given in Figure 3, 4, and 5. We have also plotted the gamma distribution with a probability density function (pdf) that minimizes the squared error to the empirical pdf for comparison. The shape and rate in the approximating gamma distribution are calculated using the approximation in 4.11 and 4.12 with $\Delta=1$ and where we truncate the sum at $i=3$, resulting in a sum of 4 gamma distributed variables that approximate the distribution of the CARMA process. The stationary distribution of the CARMA processes is simulated by discretizing the state-space equation over an interval of length $1,000,050$. We simulate on a grid with grid-size 1/10 but only consider every tenth simulated value, and we disregard the first 50 observations to approximately be in the stationary 


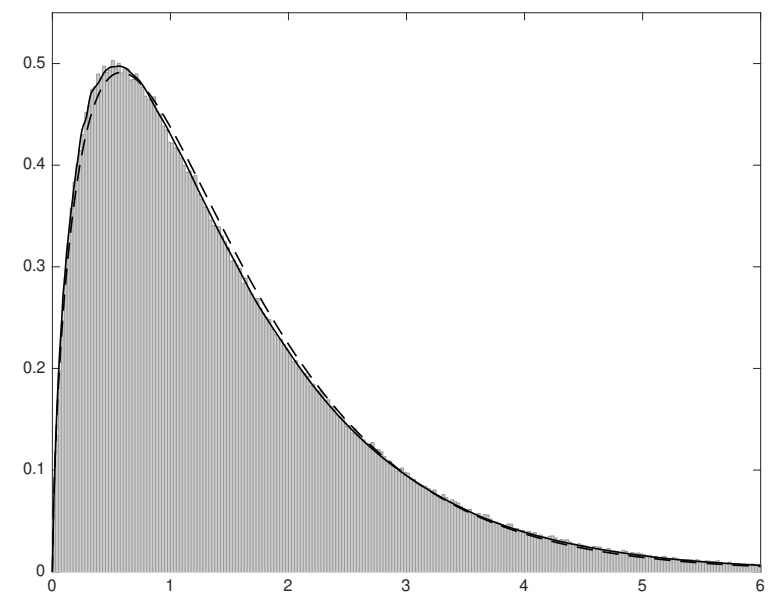

Figure 3: Histogram for simulated stationary distribution of a CARMA $(2,1)$ process in grey, pdf of sum of 4 independent gamma distributed variables (solid line), and pdf of the gamma distribution minimizing the squared error to the histogram (dotted line). Here, $\alpha_{2}=-1$.

distribution. The simulation of the stationary distribution is therefore based on $1,000,000$ observations.

We see that the sum of independent gamma distributed variables approximates the stationary distribution of the CARMA process well and that there is a significantly better fit than the gamma distribution.

\section{Acknowledgements}

We would like to thank Alexandre Brouste for providing access to the wind data analyzed in this paper. FEB gratefully acknowledges the financial support from FINEWSTOCH, funded by the Norwegian Research Council. VR gratefully acknowledges the financial support from the Danish Council for Independent Research, grant DFF-4002-00003. We would also like to thank two referees for many valuable suggestions and comments. 


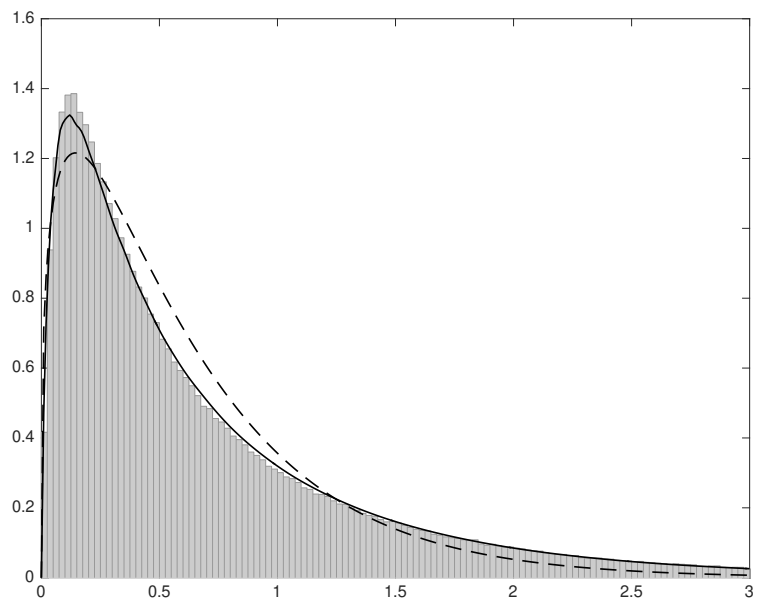

Figure 4: Histogram for simulated stationary distribution of a CARMA $(2,1)$ process in grey, pdf of sum of 4 independent gamma distributed variables (solid line), and pdf of the gamma distribution minimizing the squared error to the histogram (dotted line). Here, $\alpha_{2}=-2$.

\section{References}

[1] Andresen, A., F. E. Benth, S. Koekebakker, and V. Zakamulin (2014). The CARMA interest rate model. International Journal of Theoretical and Applied Finance 17(02), 1450008.

[2] Barndorff-Nielsen, O. E. and A. Basse-O'Connor (2011). Quasi OrnsteinUhlenbeck processes. Bernoulli 17(3), 916-941.

[3] Barndorff-Nielsen, O. E. and N. Shephard (2001). Non-Gaussian OrnsteinUhlenbeck-based models and some of their uses in financial economics. $J . R$. Stat. Soc. Ser. B Stat. Methodol. 63(2), 167-241.

[4] Basse-O'Connor, A., M. S. Nielsen, J. Pedersen, and V. Rohde (2018). Multivariate stochastic delay differential equations and CAR representations of CARMA processes. arXiv preprint arXiv:1801.04091.

[5] Bensoussan, A. and A. Brouste (2016). Cox-Ingersoll-Ross model for wind speed modeling and forecasting. Wind Energy 19(7), 1355-1365. 


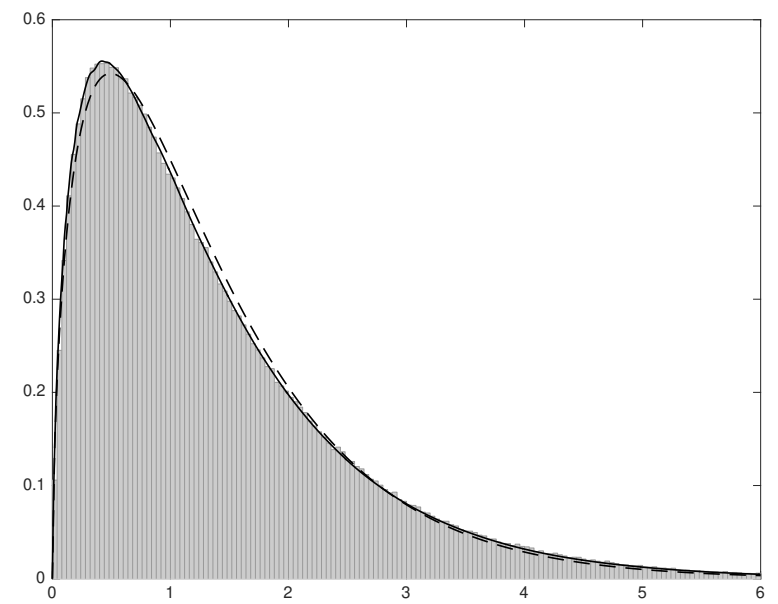

Figure 5: Histogram for simulated stationary distribution of a CARMA $(3,2)$ process in grey, pdf of sum of 4 independent gamma distributed variables (solid line), and pdf of the gamma distribution minimizing the squared error to the histogram (dotted line).

[6] Benth, F. E., J. v. Benth, and S. Koekebakker (2007). Putting a price on temperature. Scand. J. Statist. 34(4), 746-767.

[7] Benth, F. E., C. Klüppelberg, G. Müller, and L. Vos (2014). Futures pricing in electricity markets based on stable CARMA spot models. Energy Economics 44, 392-406.

[8] Benth, F. E. and A. Pircalabu (2018). A non-Gaussian Ornstein-Uhlenbeck model for pricing wind power futures. Applied Mathematical Finance 25(1), $36-65$.

[9] Benth, F. E. and A. Suess (2017). Continuous-time autoregressive movingaverage processes in Hilbert space. To appear in Abel Proceedings 2016, Springer Verlag.

[10] Billingsley, P. (1999). Convergence of Probability Measures (Second ed.). Wiley Series in Probability and Statistics: Probability and Statistics. New York: John Wiley \& Sons Inc. A Wiley-Interscience Publication. 
[11] Bondesson, L. (2012). Generalized gamma convolutions and related classes of distributions and densities, Volume 76. Springer Science \& Business Media.

[12] Brockwell, P. (2014). Recent results in the theory and applications of CARMA processes. Annals of the Institute of Statistical Mathematics 66(4), $647-685$.

[13] Brockwell, P. and A. Lindner (2013). Integration of CARMA processes and spot volatility modelling. Journal of Time Series Analysis 34(2), 156-167.

[14] Brockwell, P. and T. Marquardt (2005). Lévy-driven and fractionally integrated ARMA processes with continuous time parameter. Statist. Sinica 15(2), 477-494.

[15] Brockwell, P. J., R. A. Davis, and Y. Yang (2011). Estimation for nonnegative Lévy-driven CARMA processes. Journal of Business 83 Economic Statistics 29(2), 250-259.

[16] Brockwell, P. J. and A. Lindner (2009). Existence and uniqueness of stationary Lévy-driven CARMA processes. Stochastic Process. Appl. 119(8), 2660-2681.

[17] Brockwell, P. J. and A. Lindner (2015). Prediction of Lévy-driven CARMA processes. Journal of Econometrics 189(2), 263-271.

[18] Bru, M.-F. (1991). Wishart processes. Journal of Theoretical Probability 4 (4), 725-751.

[19] Cox, J. C., J. E. Ingersoll Jr, and S. A. Ross (1985). A theory of the term structure of interest rates. Econometrica 53(2), 385-408.

[20] Cuchiero, C., D. Filipović, E. Mayerhofer, and J. Teichmann (2011). Affine processes on positive semidefinite matrices. The Annals of Applied Probability $21(2), 397-463$.

[21] Filipovic, D. (2009). Term-Structure Models. A Graduate Course. Springer. 
[22] García, I., C. Klüppelberg, and G. Müller (2011). Estimation of stable CARMA models with an application to electricity spot prices. Stat. Model. 11(5), 447-470.

[23] Gouriéroux, C. (2006). Continuous time Wishart process for stochastic risk. Econometric Reviews 25(2-3), 177-217.

[24] Heston, S. L. (1993). A closed-form solution for options with stochastic volatility with applications to bond and currency options. The Review of Financial Studies 6(2), 327-343.

[25] Isserlis, L. (1918). On a formula for the product-moment coefficient of any order of a normal frequency distribution in any number of variables. Biometrika 12(1/2), 134-139.

[26] Jeanblanc, M., M. Yor, and M. Chesney (2009). Mathematical methods for financial markets. Springer Science \& Business Media.

[27] Karatzas, I. and S. E. Shreve (1991). Brownian motion and stochastic calculus (Second ed.), Volume 113 of Graduate Texts in Mathematics. New York: Springer-Verlag.

[28] Kučera, V. (1973). A review of the matrix Riccati equation. Kybernetika $9(1), 42-61$.

[29] Levin, J. (1959). On the matrix Riccati equation. Proceedings of the American Mathematical Society 10(4), 519-524.

[30] Marquardt, T. and R. Stelzer (2007). Multivariate CARMA processes. Stochastic Process. Appl. 117(1), 96-120.

[31] Mayerhofer, E., O. Pfaffel, and R. Stelzer (2011). On strong solutions for positive definite jump diffusions. Stochastic Processes and Their Applications 121(9), 2072-2086.

[32] Øksendal, B. (2003). Stochastic Differential Equations. Springer. 
[33] Rajput, B. S. and J. Rosiński (1989). Spectral representations of infinitely divisible processes. Probab. Theory Related Fields 82(3), 451-487.

[34] Rogers, L. C. G. and D. Williams (2000). Diffusions, Markov processes, and martingales. Vol. 1. Cambridge Mathematical Library. Cambridge: Cambridge University Press. Foundations, Reprint of the second (1994) edition.

[35] Stein, E. M. and J. C. Stein (1991). Stock price distributions with stochastic volatility: an analytic approach. The Review of Financial Studies 4(4), 727752.

[36] Todorov, V. (2009). Estimation of continuous-time stochastic volatility models with jumps using high-frequency data. J. Econometrics 148(2), 131148.

[37] Tsai, H. and K. Chan (2005). A note on non-negative continuous time processes. Journal of the Royal Statistical Society: Series B (Statistical Methodology) 67(4), 589-597.

[38] Vasicek, O. (1977). An equilibrium characterization of the term structure. Journal of Financial Economics 5(2), 177-188. 